\title{
Granulocyte colony stimulating factor in neutropenic patients with infective endocarditis
}

\author{
Birgitte Molke Borgbjerg, Doris Hovgaard, Jørn Bech Laursen, Jan Aldershvile
}

\begin{abstract}
A well known complication in the treatment of infectious endocarditis is development of neutropenia caused by treatment with antibiotics in high concentrations over long periods. Neutropenia often necessitates discontinuation of antibiotic treatment. Three patients with infectious endocarditis who developed neutropenia are reported. The patients were treated with granulocyte colony stimulating factor (G-CSF), a haematopoietic growth factor that stimulates neutrophils. G-CSF induced an immediate increase in white blood cell count, primarily neutrophils. G-CSF may be effective in ameliorating neutropenia in patients who receive antibiotics for treatment of infectious endocarditis.

(Heart 1998;79:93-95)
\end{abstract}

Keywords: granulocyte colony stimulating factor; neutropenia; endocarditis

Infective endocarditis is caused by microbial infection and is virtually always fatal if untreated. To eradicate all microorganisms from the vegetation on the endocardium, high dose, long term antibiotic treatment is required. A well known complication of $\beta$ lactam antibiotic treatment for infective endocarditis is neutropenia. ${ }^{1}$ In patients with acute leukaemia the risk of developing infection is related to the absolute neutrophil counts and depends on the degree and duration of neutropenia. ${ }^{2}$ Granulocyte colony stimulating factor (G-CSF) is a haematopoietic growth factor, a glycoprotein hormone, that promotes proliferation and differentiation of the neutrophil lineage, potentates survival, and affects mature neutrophil function. ${ }^{3}$ G-CSF has primarily been used in patients with malignant diseases to reduce chemotherapy induced neutropenia. ${ }^{4}$ It has proved efficacy in ameliorating neutropenia as well as the rate and severity of infections in chronic neutropenia. ${ }^{5}$ We report the effect of G-CSF treatment in three patients who developed leucopenia during antibiotic treatment for infectious endocarditis.
Case 1

A 42 year old woman with no previous cardiac disease was admitted to a local hospital with fever $\left(40^{\circ} \mathrm{C}\right)$ and a newly developed paresis of the left-sided extremities. One month before admission she had had two teeth extracted. Computed tomography of the brain revealed a cerebral infarct, and echocardiography showed vegetation on the aortic valve and a moderate degree of aortic regurgitation. Blood cultures revealed non-haemolytic streptococci (Streptococcus mitis) in two blood cultures after 48 hours. Intravenous penicillin G (20 million units daily) and metronidazole (1200 mg daily) were given. Temperature normalised and complete regression of neurological disturbances was observed.

The patient was transferred to our hospital for surgical evaluation five weeks after admission. Transoesophageal echocardiography confirmed the earlier findings and there was no indication for surgical valve replacement. Neutropenia (leucocytes $0.9 \times 10^{9} / 1$ and neutrophils $0.1 \times 10^{9} / 1$ ) was noticed during the next few days (fig 1, patient 1). Intravenous antibiotics had been administered for six weeks and treatment was discontinued as the infection was considered eradicated. The patient's temperature rose to $39.4^{\circ} \mathrm{C}$ the following day and erythrocyte sedimentation rate (ESR) and $C$ reactive protein (CRP) were raised (ESR $38 \mathrm{~mm}$ in the first hour; CRP $791 \mathrm{nmol} / \mathrm{l})$. Intravenous vancomycin ( $2 \mathrm{~g}$ daily), imipenem (3 g daily), and subcutaneous G-CSF (Neupogen, Roche, filgrastim $250 \mathrm{mg}$ daily) were initiated. Blood cultures were negative. On the second day of antibiotic treatment and G-CSF, imipenem was replaced by intravenous ciprofloxacin ( $800 \mathrm{mg}$ daily). Temperature was normal on the fifth day and leucocyte count had increased to $12 \times 10^{9} / 1$ resulting mainly from an increase in neutrophils $\left(8.8 \times 10^{9} / 1\right)$. Bone marrow examination revealed hypoplasia but morphologically normal cells. Alanine aminotransferase and aspartate aminotransferase were raised ( 56 and $57 \mathrm{U} / 1$, respectively). Antibiotics were given for 10 days. The patient was discharged from the hospital and eight months later was well. 
Case 2

A 30 year old man was admitted to a local hospital with remittent low grade fever $\left(38^{\circ} \mathrm{C}\right)$ for two months. The patient suffered from fatigue,
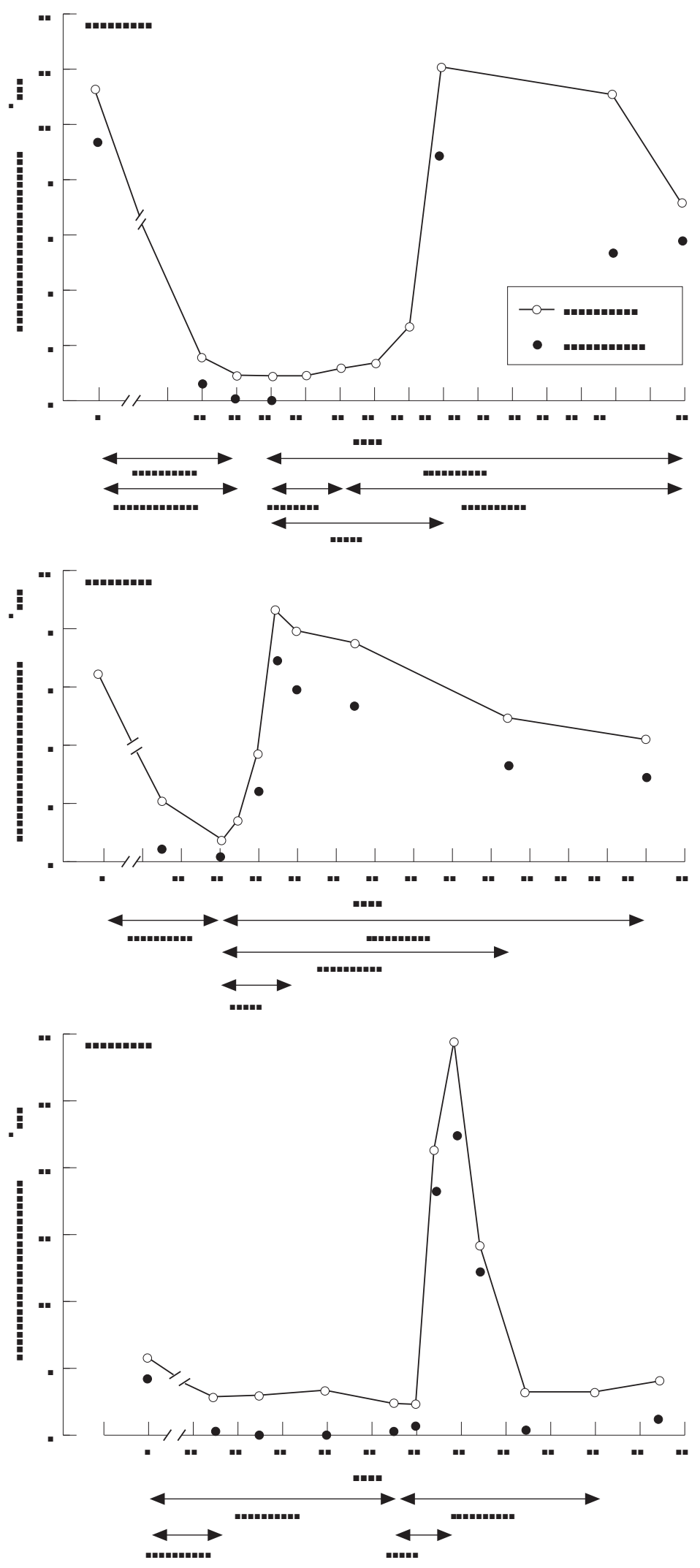

Figure 1 Changes in leucocyte and neutrocyte counts in three patients receiving G-CSF and antibiotic treatment for infectious endocarditis. malaise, weight loss, and minor arthritis symptoms. He had experienced five episodes of visual disturbances lasting for about five minutes. Echocardiography revealed aortic regurgitation and left ventricular volume overload. Blood cultures grew non-haemolytic streptococci ( $S$ viridans) in two cultures (repeated three times) after 72 hours. White blood cell count was normal. Intravenous penicillin $G$ (16 million units daily) was initiated. After one week his temperature dropped to normal but relapsed within two weeks. The patient was transferred to our hospital with a temperature of $39.5^{\circ} \mathrm{C}$, petechiae, splinter haemorrhages, and exanthem. Echocardiography revealed bicuspid aortic valves with minor vegetations, severe aortic regurgitation, and subvalvar aneurysm. Leucopenia had developed on the day of transfer (leucocytes $0.9 \times 10^{9} / 1$, neutrophils $0.1 \times 10^{9} / 1$ ) (fig 1, patient 2). Intravenous penicillin was stopped and replaced by intravenous vancomycin (2 g daily) and gentamicin (initially $140 \mathrm{mg}$ and later adjusted according to serum concentration), and G-CSF (300 mg daily) subcutaneously was initiated. Over the following two days white cell count raised (leucocytes $8.6 \times 10^{9} / 1$, neutrocytes $6.9 \times 10^{9} / 1$ ), temperature fell, and G-CSF was stopped. A Ross operation was performed one week later to correct the valve insufficiency. Microbiological examination of the removed bicuspid valve did not reveal growth of any bacteria. The patient was well without symptoms five months after discharge.

\section{Case 3}

A 74 year old woman underwent aortic valve replacement (biological tissue valve; Carpentier-Edwards 21) because of aortic stenosis. Four months after the operation the patient was admitted to the hospital with a temperature of up to $40.0^{\circ} \mathrm{C}$. One week before admission she had undergone a dental extraction because of infection. Antibiotics had been taken prophylactically as prescribed by the department. No clinical signs suggesting infectious endocarditis was noted on admission. Cross sectional echocardiography showed no vegetation but a prevalvar aortic pressure gradient $\quad(50-60 \mathrm{~mm} \mathrm{Hg})$ was diagnosed. Laboratory findings revealed an increased ESR (60 $\mathrm{mm}$ in the first hour), and CRP (1277 nmol/1), normal white blood cell count $\left(6.1 \times 10^{9} / 1\right)$, and thrombocytopenia $\left(64 \times 10^{9} / 1\right)$ (fig 1, patient 3 ). Blood cultures grew nonhaemolytic streptococci ( $S$ anginosus) after 48 hours in three cultures (twice). Intravenous penicillin G (16 million units daily) and netilmicin (240 mg daily) were initiated immediately. Temperature dropped and blood culture became negative. Three weeks later white blood cell count had fallen to $2.6 \times 10^{9} / 1$ (neutrocytes $0.4 \times 10^{9} / 1$ ) and ESR was still raised (54 $\mathrm{mm}$ in the first hour). Penicillin was replaced by vancomycin ( $2 \mathrm{~g}$ daily) and G-CSF (300 mg daily subcutaneously) was initiated. White cell count increased to $29 \times 10^{9} / 1$ within two days of G-CSF treatment, almost entirely 
from an increase in neutrophils (neutrocytes $\left.22 \times 10^{9} / 1\right)$. The patient's temperature fell below $37^{\circ} \mathrm{C}$. She was well one month after discharge.

\section{Discussion}

G-CSF has been shown to ameliorate chemotherapy induced neutropenia, and therefore the incidence of fever and associated morbidity in patients with malignant diseases. ${ }^{4}$ The effect of G-CSF is because of an initial release of neutrophils from the bone marrow followed by an increased production of neutrophil progenitor cells, an increased amplification in the maturation compartment in the bone marrow, and early release of mature neutrophils. ${ }^{6}$ The neutrophils have normal mobility and bactericidal activity indicating functional competency. ${ }^{7}$ Enhanced neutrophil function by G-CSF has been shown to improve in vitro bactericidal activity. ${ }^{8}$ Animal experiments suggest that G-CSF is useful as an adjuvant to antibiotic treatment in leucopenic patients. ${ }^{9}$ No major clinical trials have applied G-CSF as adjunctive treatment to antibiotics in infectious diseases. So far only two patients with infective endocarditis have been reported to receive G-CSF treatment for neutropenia. ${ }^{10}{ }^{11}$ In the present report three patients with infectious endocarditis developed neutropenia and received G-CSF treatment. During severe infections it is well known that neutropenia can appear either secondary to the infection per $\mathrm{se}^{12}$ or because of antibiotic treatment. ${ }^{1}$ In all three patients reported here, G-CSF induced an immediate increase in white blood cell count, primarily neutrophils and, after discontinuation, neutropenia reappeared in one patient (case 3). Neutropenia after G-CSF treatment has been observed previously and is thought to be of shorter duration and less profound. ${ }^{13}$ Treatment with G-CSF was only necessary for a few days $(2-5)$ in contrast to the previous two case reports where they used G-CSF for five months and 14 days, respectively. ${ }^{10} 11$ Also a change in antibiotic treatment might account for some of the rise in total leucocyte count. Treatment with penicillin, an antibiotic known to induce neutropenia, ${ }^{1}$ was stopped before G-CSF treatment. A change in antibiotic treatment was also noted in the two previous case reports..$^{11}$ We observed a slight increase in aspartate and alanine aminotransferases after G-CSF treatment in one of the patients, but no other side effects such as influenza-like symptoms or fever. Neither did we observe any vascular complications as reported in other studies. ${ }^{1314}$ However, other factors could have influenced these studies, such as malignant disease, chemotherapy, and atherosclerosis. Generally G-CSF was well tolerated in the three patients.

In conclusion, G-CSF seems effective in ameliorating neutropenia in patients who receive antibiotics to treat infectious endocarditis.

We are indebted to Dr Ernö Gutschik for his expertise in microbiology.

1 Olaison L, Alestig K. A prospective study of neutropenia induced by high doses of beta-lactam antibiotics. F Antimicrob Chemother 1990;25:449-53.

2 Bodey G, Buckley M, Sathe Y, Freireich E. Quantitative relationships between circulating leucocytes and infection in patients with acute leucemia. Ann Intern Med 1996;64: in patients

3 Groopman J, Molina J, Scadden D. Hematopoietic growth factors. $N$ Engl $\mathcal{F}$ Med 1989;321:1449-59.

4 Pettengell R, Howard G, Radford J, Dealin D, James P, Wilkinson P, et al. Granulocyte colony-stimulating factor to prevent dose-limiting neutropenia in non-Hodgkin's lymphoma: a randomized controlled trial. Blood 1992;80: $1430-6$

5 Dale D, Bolyard A, Hammond W. Cyclic neutropenia: natural history and effects of long-term treatment with recombinant human granulocyte colony-stimulating factor. Cancer Invest 1993;11:219-23.

6 Lord B, Bronchud M, Owens S, Chang J, Howell A, Souza $\mathrm{L}$, et al. The kinetics of human granulopoiesis following treatment with granulocyte colony-stimulating factor in vitro. Proc Natl Acad Sci 1989;86:9499-503.

7 Bronchud M, Scarffe J, Thatcher N, Crowther D, Souza L, Bronchud M, Scarffe J, Thatcher N, Crowther D, Souza L,
Alton N, et al. Phase I/II study of recombinant human glton $\mathrm{N}$, et al. Phase $\mathrm{I} / \mathrm{II}$ study of recombinant human granulocyte colony stimulating factor in patients recieving
intensive chemotherapy for small cell lung cancer. $\mathrm{Br} f$ intensive chemotherapy

8 Roilides E, Walsh T, Pizzo P, Rubin M. Granulocyte colonystimulating factor enhances the phagocytic and bactericidal activity of normal and defective human neutrophils. F Infect Dis 1991;163:579-83.

9 Matsumoto M, Matsubara S, Yokota T. Effect of combination therapy with recombinant human granulocyte colonystimulating factor (rG-CSF) and antibiotics in neutropenic mice unresponsive to antibiotics alone. 7 Antimicrobial Chemot 1991;28:447-53.

10 Engle M, Lang S, Edelson P. Endocarditis with aneurism involving an aortic homograft used to correct a truncus arteriosus: medical-surgical salvage. Br Heart $f$ 1992;67: 409-11.

11 Hanazono Y, Miyazono K, Tsuchimochi H, Yamaoki K, Miagawa $\mathrm{K}$, Hirai $\mathrm{H}$, et al. Treatment of infective endocar-
ditis with granulocyte colony-stimulating factor. $f p n \mathcal{F} \mathrm{Med}$ ditis with granu

12 Goya T, Torisu M, Doi F, Yoshida T. Effects of granulocyte colony stimulating factor and monobactam antibiotics (Aztreonam) on neutrophil functions in sepsis. Clin Immun Immunpathol 1993;9:278-84.

13 Lindemann A, Herrmann F, Oster W, Haffner G, Meyenburg W, Souza L, et al. Hematologic effects of recombinant human granulocyte colony stimulating factor in patients with malignancy. Blood 1989;74:2644-51.

14 Lindemann A, Rumberger B. Vascular complications in patients treated with granulocyte colony-stimulating factor (G-CSF) [letter]. Eur f Cancer 1993;29:2338-9. 